\title{
Gene-by-Environment Interactions in Human Individual Differences
}

Jaime Derringer $^{* 1}$, Jonathan Livengood ${ }^{2}$, Daniel A. Briley ${ }^{1}$

Submitted draft as of 23 April 2019

Additional materials available at https://osf.io/mc5e2/

${ }^{1}$ Department of Psychology, University of Illinois at Urbana-Champaign

${ }^{2}$ Department of Philosophy, University of Illinois at Urbana-Champaign

* Corresponding Author: Jaime Derringer, jderr@illinois.edu 


\begin{abstract}
The existence of gene-by-environment interactions (GxE) influencing human individual differences is likely, given both evidence from non-human animal models and evolutionary theory as applied to human development. However, there is no consistent evidence in the current psychological literature of any specific, replicable measured genotype-by-environment interaction. This project will systematically evaluate genome-wide GxE across a range of individual differences and environmental exposures, applying the Registered Report model to secondary data analysis of the UK Biobank. We will estimate the genome-wide distribution of GxE effect sizes, evaluate the shapes of identified interactions (diathesis-stress, vantage-sensitivity, and cross-over), and identify genetic variants that show evidence of generalized plasticity.
\end{abstract}

Keywords: gene-environment interaction; GWAS; body size; cognitive ability; personality 


\section{Gene-by-Environment Interactions in Human Individual Differences}

The presence of gene-by-environment interactions (GxE) influencing human individual differences is highly likely, given both evidence from non-human animal models (Saltz et al., 2018) and evolutionary theory as applied to human development (Belsky,

Bakermans-Kranenburg, \& Van IJzendoorn, 2007). However, there is no consistent evidence in the current human psychological literature of any specific, replicable measured genotype-by-environment interaction. The vast majority of the existing measured genotype-by-environment interaction literature focuses heavily on a small number of candidate genes. Previously investigated candidate genotype-by-environment interactions have a rocky history of failures to replicate (Haberstick et al., 2014; Border et al., 2019) and dueling meta-analyses that come to different conclusions from the same literatures (Risch et al., 2009; Karg et al., 2011). The candidate gene literature is characterized by limited sample sizes (Duncan \& Keller, 2011), flawed statistical approaches (Keller, 2014), and a lack of systematic evaluation of effects (Dick et al., 2015).

Here, we plan to address these limitations by taking a broad and consistent look across four phenotypes (neuroticism, risk taking, fluid intelligence, and body mass index) and four environments (stressful life events, smoking status, regional socioeconomic status, and urban-rural residency). In a large, pre-existing study with available phenotypes, environments, and genome-wide data, we will estimate the distribution of GxE effect sizes, evaluate the shapes of identified interactions (diathesis-stress, vantage-sensitivity, and cross-over), and test whether any single nucleotide polymorphisms (SNPs, pronounced "snips"; individual rungs on the ladder of DNA that vary between people) show evidence of generalized plasticity, in the form of GxE effects appearing across multiple phenotypes or environments.

\section{What Effect Sizes Can We Expect?}


In preparing to identify replicable GxE effects, we may learn from the recent history of the development of successful, standardized pipelines for discovery of novel effects of SNPs through the genome-wide association study (GWAS) method. In a GWAS, millions of individual genetic variants are each tested for association with a phenotype, evaluated against a multiple testing corrected threshold for statistical significance of $p<5 \times 10^{-8}$. Early on, the absence of large, statistically significant effects led some to wonder about the apparently missing heritability (Maher, 2008). However, as sample sizes increased, replicable associations began to emerge (such as for height, Allen et al., 2010; schizophrenia, Ripke et al., 2011; and educational attainment, Rietveld et al., 2013). It became clear that, regardless of the phenotype, the effects the field was searching for were incredibly small (Chabris et al., 2015). For example, the largest identified individual SNP association with educational attainment was the equivalent of a correlation of 0.01 (Rietveld et al., 2013). In GWASs of complex behavioral phenotypes, such as personality and cognitive ability, progress in discovering the many small genetic effects underlying a trait's heritability required exceptionally large sample sizes.

Progress toward genome-wide GxE has been slowed by uncertainty with respect to the data requirements for executing a 'successful' study, in consequence of uncertainty regarding anticipated effect sizes. Rather than focus on specific effects, we may address this question generally by evaluating, genome-wide across a range of phenotypes and environments, the overall distribution of GxE effect sizes. Estimating genome-wide distributions of GxE effect sizes would allow us to develop a framework for anticipating the frequency and size of GxE effects that researchers might reasonably expect going forward.

\section{Is There a Common Interaction Shape?}

Much prior theoretical work has focused on descriptions of possible "shapes" of interactions between genes and environment (Roisman et al., 2012). In this paper we will let the 
shape of an interaction refer to one of three forms: diathesis-stress, vantage-sensitivity, and cross-over. Under diathesis-stress, effects of the genotype are strongest in negative environments. Diathesis-stress is a common theorized model in psychopathology (Caspi et al., 2002; Caspi et al., 2003). Under vantage-sensitivity, effects of the genotype are strongest in positive environments. Vantage-sensitivity has been implicated in the effect of socioeconomic status on the heritability of cognitive ability, where US samples appear to show greater heritability at higher levels of SES (Tucker-Drob \& Bates, 2016). In cross-over interactions, including the dandelion/orchid hypothesis (Boyce \& Ellis, 2005), the "risk" allele differs depending on whether the environment is positive or negative.

The genome-wide search for individual GxE effects is complicated by the fact that interactions might have any of these different potential shapes, since differently shaped interactions will have different relationships with observed GWAS effects and different associated p-values in null hypothesis significance testing. Both diathesis-stress and vantage-sensitivity interactions would be expected to have non-zero SNP effects in a standard GWAS. Cross-over interactions would not be expected to show consistent SNP effects in a GWAS, and are described by larger beta weights in a regression (and consequently lower p-values) compared to diathesis-stress or vantage-sensitivity effects. Thus, a uniform p-value threshold for declaring statistical significance would relatively over-identify cross-over effects and under-identify diathesis-stress and vantage-sensitivity effects (Dick et al., 2015; Hsu et al., 2012).

Several recent papers have attempted to circumvent potentially limited - or unknown statistical power to detect individual genetic variant GxE effects by applying a polygenic score approach (eg. Celis-Morales, et al., 2017; Peyrot et al., 2018; Barcellos, et al., 2018), in which a linear combination of SNP effects identified from and weighted by GWAS results is tested for 
moderation by an environmental exposure. Implicitly, this approach presumes (a) that the underlying shape of interactions is consistent across variants and (b) the shape is diathesis-stress or vantage-sensitivity, rather than cross-over, which would not be expected to show consistent SNP effects in a GWAS. No compelling theoretical arguments or empirical studies support the claim that either of those two conditions is met. And if either one of them is not met, then individual-variant level GxE may be present even in the absence of GWAS-derived polygenic score moderation.

\section{Do Some SNPs Show Moderation Across Phenotypes and Environments?}

The vast majority of empirical approaches to GxE focus on a single phenotype-environment combination, without elucidating whether that particular combination is expected to be representative of a broader set of theorized relationships (Dick et al., 2015; Duncan \& Keller, 2011). Conversely, the theoretical literature addressing the potential for GxE effects tends to discuss such influences broadly, speaking of positive and negative environments and also positive and negative phenotypes (Belsky, Bakermans-Kranenburg, \& Van IJzendoorn, 2007). The main effects literature is much clearer at this point. For the most part, shared genetic influences tend to underlie observed phenotypic correlations, whether such estimates are made on the basis of inferred heritability in the context of twin and family studies (Kendler et al., 2011) or GWAS-estimated SNP effects (Bulik-Sullivan et al., 2015). These patterns of observed genetic overlap may arise from a variety of mechanisms, including but not limited to multidetermined phenotypes, pleiotropy, and gene-environment interplay (Briley, Livengood, \& Derringer, 2018). Rather than target a specific combination of phenotype and environment a priori for GxE investigation, we may instead ask to what extent individual SNPs display evidence of plasticity; that is, whether certain SNPs are more sensitive across broad classes of environmental inputs. 


\section{The Current Study}

We propose to systematically evaluate a range of genome-wide GxE environment-phenotype combinations. Although individual GxE effects may be identified and replicated from our approach, characterization of singular GxE effects is not our primary goal. Rather, we seek to transparently and broadly describe the observed structure of genotype-by-environment interactions, to serve as a fundamental basis for the field in developing a holistic perspective on GxE effects.

Although much is known and being discovered about the forms and structures of main effects of genetic variants on human individual differences, nearly everything remains unknown about what plausible genotype-by-environment interactions would look like (Briley et al., 2019). To that end, this project seeks to address three research questions that are central to understanding GxE effects in humans:

(RQ1) Whether the distribution of genome-wide GxE effects is different from the distribution of GWAS effects;

(RQ2) Whether diathesis-stress, vantage-sensitivity, and cross-over effects occur with equal frequency; and

(RQ3) Whether moderated SNPs show consistent evidence of plasticity across combinations of phenotypes and environments.

\section{Methods}

We will conduct secondary analysis of UK Biobank data (https://www.ukbiobank.ac.uk/; Bycroft et al., 2018). The UK Biobank has available genetic, phenotypic, and environmental measures on around 190,000 to 480,000 individuals, depending on the specific phenotype-environment combination (see below for target measures). Access to the UK Biobank data requires specific application. None of the authors on this proposal have ever had 
direct access to UK Biobank data, and we will not access any data until after a Stage 1 Registered Report has been reviewed and accepted in principle.

\section{Participants}

The UK Biobank is a population sample of mid-life to older adult residents in the United Kingdom. The average age was 56.5 years at baseline (range $=37-73$ ), with $54.4 \%$ self-identifying as female, and $45.6 \%$ self-identifying as male. Participants with available genotype data (the downloadable version of which has been pre-cleaned according to standard quality control protocols; Bycroft et al., 2018) as well as data available on at least one of the target phenotypes and one of the target environments, and have no relatives 3rd degree of genetic relatedness or closer in the resulting dataset. As previously described (Bycroft et al., 2018, supplementary information S 3.7.4), related individuals will be identified as those pairs with pre-estimated kinship coefficients $\geq 0.44$. Participants will be selected for exclusion such that the smallest number of participants is excluded to break up estimated relatedness networks, using the igraph package in R (Csardi \& Nepusz, 2016). Prior to analysis, the data will be split randomly into discovery ( $80 \%$ of the full sample) and replication (the remaining $20 \%$ ) samples. Each test with include all participants will full data available on the required set of variables (covariates and the specific combination of phenotype, environment, and genotype). The exact number of participants is expected to vary across tests depending on missingness patterns for each combination of phenotype, environment, and genotype; the total $\mathrm{N}$ will be reported for each test.

\section{Measures}

Rather than focusing on a particular phenotype-environment relationship, we seek to broadly characterize genome-wide GxE effects across a variety of phenotype-environment combinations. To that end, we have selected four phenotypes (risk taking, body mass index, 
neuroticism, and fluid intelligence) and four environments (smoking status, stressful life events, urban-rural residential location, and regional socioeconomic status), which will be fully crossed for a total of 16 genome-wide GxE studies on which to base our conclusions. Phenotypes and environments were identified from pre-existing variables in UK Biobank. Details and descriptive statistics for each are available at:

https://biobank.ctsu.ox.ac.uk/crystal/field.cgi?id=DATA_FIELD; the data field identifier is listed for each variable described below.

\section{Phenotypes.}

Risk taking (data field = 2040) was assessed as endorsement of a single item, "Would you describe yourself as someone who takes risks?". It is available on 501,643 participants, with an endorsement rate of $26.8 \%$.

Body mass index $(\mathrm{BMI}$; data field $=21001)$ was calculated as weight $(\mathrm{Kg})$ divided by height squared $\left(\mathrm{m}^{2}\right)$. Weight and height were measured in-person. BMI is available on 499,520 participants, with a mean of $27.4(\mathrm{SD}=4.8$, range $=12.1-74.7)$.

Neuroticism (data field $=20127$ ) was assessed as a count of how many of 12 items a participant endorsed, including: "Does your mood often go up and down?"; "Do you ever feel ‘just miserable' for no reason?”; "Are you an irritable person?"; “Are your feelings easily hurt?”; “Do you often feel 'fedup'?”; “Would you call yourself a nervous person?”; "Are you a worrier?”; “Would you call yourself tense or 'highly strung'?"; "Do you worry too long after an embarrassing experience?”; “Do you suffer from 'nerves'?"; "Do you often feel lonely?"; "Are you often troubled by feelings of guilt?". It is available on 401,596 participants, with a mean of 4.1 (SD $=3.3$, range $=0-12)$.

Fluid intelligence (data field $=20016$ ) was assessed as number of questions answered correctly on a test of verbal and numeric reasoning. It has previously been shown to correlate 
with other measures of cognitive function and load strongly on a general intelligence factor in the same UK Biobank sample (Lyall et al., 2016). It is available on 197,602 participants, with a mean of $6.1(S D=2.2$, range $=0-13)$.

\section{Environments.}

Smoking status (data field $=20116$ ) is categorized into current, former, and never smokers. It is available on 501,659 participants, with $38.4 \%$ former smokers and $11.0 \%$ current smokers. Although smoking status is not a common environmental target for GxE investigation, smoking is robustly associated with genome-wide alterations of methylation (Zielinger et al., 2013), which has been suggested as a possible mechanism for GxE effects (Abdolmaleky et al., 2004; Klengel \& Binder, 2015).

Regional socioeconomic status (SES; data field = 189) was assessed using the Townsend deprivation index, a regional aggregation of unemployment, (non-)car ownership, (non-)home ownership, and household overcrowding (Townsend, 1987). This regional assessment of SES is strongly genetically correlated with individual measures of SES, such as income, although the phenotypic correlation is moderate (Hill et al., 2016). We elect to use the aggregate regional assessment of SES over available measures of household or individual income to avoid measurement uncertainty which may be introduced by individual variation on, for example, retirement status or household size (e.g. dual versus single earners), and in light of substantial prior evidence that aggregate regional SES is a valid predictor of many measures of morbidity and mortality (Smith et al., 2001). Regional SES is available on 501,914 participants, with a mean of $-1.3(S D=3.1$, range $=-6.3-11.0)$.

Stressful life events (SLE; data field $=6145)$ will be estimated as a count of stressors experienced in the past two years, out of six assessed: "Serious illness, injury or assault to yourself", "Serious illness, injury or assault of a close relative", "Death of a close relative", 
"Death of a spouse or partner", "Marital separation/divorce", and "Financial difficulties". It is available on 498,705 participants, with $45.4 \%$ of participants reporting having experienced at least one stressor in the past two years.

Urban-Rural residential area (data field $=20118$ ) will be derived from a categorical variable representing the density and accessibility of residential postcodes. It will be dichotomized into urban (postcodes with populations of 10,000 people or more; represented in the data field as codes $1,5,11$, and 12 ;

http://biobank.ndph.ox.ac.uk/showcase/docs/UrbanRural.pdf) versus less dense (hereafter referred to as 'rural'). Urban-Rural residential area is a popular target for gene-environment interaction models of immune system phenotypes, such as asthma and allergies, as a potential indicator of differential exposure to pollution, pathogens, and allergens (Schröder et al., 2015; Van Os, Rutten, \& Poulton, 2008). It is available on 497,485 participants, with $86.2 \%$ living in urban areas.

\section{Genotypes.}

Imputed genotypes are available on 487,320 participants. Restricting to autosomal SNPs with minor allele frequencies (MAFs; the frequency of the less common allele) of at least $1 \%$ that were directly genotyped or imputed with an imputation quality score of at least 0.3 will yield a dataset of approximately 10 million SNPs available for analysis. The genetic data include 40 pre-estimated ancestry principal components (PCs, data field $=22009)$, for use as covariates to control for population stratification (Bycroft et al., 2018).

\section{Statistical Analyses}

A draft analytic script for applying the below described two-step procedure to evaluate genome-wide GxE effects is available at: https://osf.io/mc5e2/. Complete results of all analyses will be made publicly available at the same osf project. 


\section{Model for genome-wide gene-by-environment interaction tests.}

Continuous phenotypic and environmental measures will be converted to Z-scores, and environmental measures will be oriented such that positive values indicate a presumptively more beneficial environment (high SES, low SLE). Risk taking will be coded 0/1, with 1 indicating endorsement. Residential location will be coded as urban $=-1$, non-urban $=+1$, as urban residency has been associated with higher rates of mood and substance use disorders (Vassos et al., 2016), and higher BMI in the UK Biobank, specifically (Flint \& Cummins, 2016). Smoking status will be coded as $-1=$ never smoker, $0=$ former, $1=$ current.

We will apply a method for establishing statistical significance of individual GxE interactions in the context of potentially different interaction shapes (cross-over versus vantage sensitivity or diathesis-stress) that relies on having GWAS results available for both the phenotype and the environment. Those GWAS results may come from the same sample in which the GxE terms will eventually be estimated, without resulting in inflation of the expected false positive rate (Hsu et al., 2012). Therefore, we begin in the $80 \%$ discovery sample by conducting:

a. a GWAS for each of the four phenotypes;

b. a GWAS for each of the four environments; and

c. a genome-wide GxE association study for each of the 16 possible phenotype-by-environment combinations.

All models will be fit as regressions in $\mathrm{R}$ ( $\mathrm{R}$ Core Team, 2018). Estimates of effects on categorical measures (GWAS and GxE of the risk taking phenotype; GWASs of the urban-rural and smoking status environments) will be fit to logistic models (ordinal, in the case of smoking status); all other phenotype GWASs and GxE and environment GWASs will be estimated as linear models. All GWASs (of phenotypes and environments) will include as covariates the main 
effects of age, sex, and 40 ancestry PCs. GxE models will further include as covariates the main effects of the target SNP and the environmental variable, as well as interactions between the age, sex, and PC covariates and the target SNP and environment (Keller, 2014).

Evaluations relevant to each of our three research questions will further require estimates of observed (1) effect sizes of GWAS effects and GxE effects, and (2) the location (in terms of environment level) of the intersection of the simple slopes for each genotype, for classifying shapes of GxE interactions that are subsequently identified as statistically significant. Both the effect sizes and the intersection location will be saved alongside the usual resulting regression parameter estimates. In order to be comparable across measures and models, the target effect size for evaluation will be the $r^{2}$ for the target effect (either SNP main effect in the phenotype and environment GWASs, or the GxE term in the interaction models). $\mathrm{R}^{2}$ (or pseudo- $r^{2}$, in models of dichotomous measures) for each target term will be estimated as the change in model $\mathrm{R}^{2}$ when comparing the covariates only model (age, sex, PCs in the GWAS; age, sex, PCs, SNP, environment, and all combinations of age/sex/PC x SNP/environment in the GxE models) to the full model (where the only difference is the addition of the target term, either the SNP effect or the GxE term). The 95\% confidence interval for the simple slopes intersection location will be estimated and saved for every GxE test using the pequod package in R (Mirisola \& Seta, 2016). However, this information will only be interpreted for those GxE effects that meet the sliding criteria for statistical significance, as described below in RQ2.

\section{(RQ1) Estimating the distribution of GxE effect sizes.}

In order to ground our interpretation of observed GxE effects in the existing statistical genetic literature, we will compare the distribution of GxE effects to the distribution of GWAS effects on phenotypes and environments using the non-parametric Kolmogorov-Smirnov test for equivalent distributions (Marsaglia, Tsang, \& Wang, 2003). The omnibus test, in which the 
distributions of effects will be aggregated across phenotypes and environments, will be evaluated compared to a threshold for statistical significance of $p<0.05$. Additional exploratory tests will be conducted for each of the 16 specific phenotype-by-environment crosses, which will be evaluated compared to a threshold for statistical significance of $p<0.05 / M_{\text {eff, }}$ where $M_{\text {eff }}$ is the effective number of tests taking into account the expected non-independence (that is, non-zero correlation) among the phenotypes and environments (Derringer, 2018). In this case, $M_{\text {eff }}$ (total) will be estimated as $M_{\text {eff }}$ (phenotypes) $\times M_{\text {eff }}$ (environments). If the variables are uncorrelated, $\mathrm{M}_{\text {eff }}$ correction approaches the equivalent of Bonferroni correction, which in this case would be $p<0.05 / 16=0.003125$.

\section{(RQ2) Interaction shapes across identified GxE effects.}

To account for the statistical properties of different forms of GxE, the estimated GxE effects will be evaluated against a binning approach of graduated significance thresholds. To identify individual SNPs whose GxE effects meet statistical significance, the total alpha (0.05) will be distributed across individual tests according to GWAS evidence of SNP associations with either the phenotype or the environment, which accounts for different statistical patterns expected to result from cross-over versus diathesis-stress or vantage-sensitivity effects. SNPs that show GWAS evidence of association with either the phenotype or the environment are more likely to be of the diathesis-stress or vantage-sensitivity forms, which would result in smaller GxE regression terms, and consequently larger p-values, and so are ranked more highly and allotted a more generous distribution of the total alpha. Conversely, cross-over effects would not manifest a GWAS association with either the phenotype or the environment, and would be described using relatively larger GxE regression terms and lower $p$-values, and therefore are allocated more stringent distributions of the total alpha. Briefly, SNPs are sorted by increasing p-values from the lower of their phenotype or environmental GWAS effect p-value. 
SNPs are then binned into increasingly large groups. Each group receives a partial distribution of the total alpha, which is split equally among all SNPs within that group. The p-value for each GxE term is then evaluated against its rank-distributed per-test alpha threshold (see 'Cocktail-II' procedure in Hsu et al., 2012). This approach is demonstrated in the example R script available at https://osf.io/mc5e2/.

For each GxE effect that is identified as statistically significant, the shape will be evaluated using the $95 \%$ confidence interval for value of the environment at which the simple slopes lines for the genotypes are estimated to intersect. If the confidence interval is entirely greater than zero, the effect will be classified as diathesis-stress; less than zero, vantage-sensitivity; overlapping with zero, cross-over. The observed frequency of each shape will be reported for each of the phenotype-environment crosses, as well as overall. The omnibus test of whether each of the three shapes shows up in equal frequency across all 16 phenotype-environment crosses will be evaluated against $x^{2}(2)$ using a critical $p$-value threshold of 0.05 . Further, exploratory tests will evaluate whether the frequencies of the three shapes are unequal within each of the 16 phenotype-environment crosses, evaluated against $x^{2}(2)$ using a critical p-value threshold of $0.05 / \mathrm{M}_{\text {eff, }}$ as described above in RQ1.

Power analysis. All power analyses were conducted using the pwr package in $\mathrm{R}$ (Champely, 2018). Given a minimum anticipated total sample size of $N=150,000$, yielding a minimum $80 \%$ discovery sample size of 120,000 , and approximately 10 million SNPs to be tested, we will have $90 \%$ power to detect effect sizes in the discovery sample equivalent to $r^{2}=$ $0.01 \%$ (at $p<0.005$ in the top ranked group) to $0.07 \%$ (at $p<4.5 \times 10^{-15}$ in the bottom ranked group). However, many phenotype-environment crosses are expected to have substantially larger available sample sizes. For example, for the majority of crosses where we may expect a total sample size of at least 400,000 participants, yielding a $80 \%$ discovery sample of $\mathrm{N}=$ 
320,000 . In these cases, we would have $90 \%$ power to detect effect sizes equivalent to $r^{2}=$ $0.005 \%$ (at $p<0.005$ in the top ranked group) to $0.03 \%$ (at $p<4.5 \times 10^{-15}$ in the bottom ranked group). These are reasonable anticipated effect sizes if the magnitude of GxE effects is similar to SNP effects observed in GWASs of complex human traits (Briley et al., 2019).

Replication. Individual SNPs with GxE effects identified as statistically significant in the $80 \%$ discovery sample will be tested, using the same regression model, in the set-aside $20 \%$ replication sample. The critical threshold for declaring a replication GxE term statistically significant will be set at 0.05 divided by the effective number of independent tests, taking into account linkage disequilibrium (LD, the tendency for physically proximal genetic variants to be correlated) among the identified SNPs (Nyholt, 2004). Given a replication sample size of at least $30,000(20 \%$ of minimum anticipated total $N \sim 150,000)$, if there were 10 independent 'hits' we would have $90 \%$ power to replicate GxE effects of at least $r^{2}=0.06 \%$ at $p<0.005$. For larger available replication sample sizes (e.g. 80,000 ) where we might expect more SNPs to cross the threshold for statistical significance in the larger-scale discovery sample (e.g. in the case of a minimum anticipated total $\mathrm{N}$ of 400,000 ), if there were 100 independent 'hits' we would have $90 \%$ power to replicate GxE effects of at least $r^{2}=0.03 \%$ at $p<0.0005$.

(RQ3) Identifying SNPs with cross-phenotype, cross-environment plasticity.

For each SNP, the observed count of statistically significant interactions across all 16 phenotype-environment crosses will be evaluated against $x^{2}(1)$, where the expected count is the average number of interactions observed for all SNPs genome-wide. This will result in a single omnibus $p$-value per each tested SNP, similar to what would result from a single-phenotype GWAS. Statistical significance for individual SNPs here will be set at the standard threshold for GWAS significance, $p<5 \times 10^{-8}$ (or the equivalent of a Bonferroni correction of an alpha of 0.05 for one million independent tests). Results will be summarized using a Manhattan plot, where 
each SNP is plotted along the $x$-axis with regard to chromosomal location and along the $y$-axis at -log10(p-value), as well as in a QQ-plot, where sequential observed p-values are plotted against the expected null distribution of p-values. The overall pattern of inflation of statistically significant $p$-values versus what would be expected under the null will be evaluated using the genomic inflation factor ( $\lambda$; Yang et al., 2011), with a value over 1 taken as an indication of more cross-phenotype/cross-environment moderation than would be expected by chance alone, given the degree of multiple testing.

\section{Robustness checks.}

Although statistical genetic methods have arrived at a standardized method of handling population stratification (ancestry PCs), there remain lingering questions about how adequately these approaches handle subtle, non-random, unmeasured confounds, even within putatively homogeneous samples (Berg et al., 2019; Sohail et al., 2018). To address this, the vast majority of genetic research has been limited to artificially restricted samples, primarily those of European ancestry (Mills \& Rahal, 2019). This has led to a huge discrepancy in information available from European populations compared to non-European populations. To partially address concerns about residual population stratification, as well as to provide estimates for samples that are comparable to those that have historically dominated statistical genetic research, we will run through the entirety of the analyses described above in the majority (84\%) subsample of individuals in the UK Biobank identified as having relatively homogeneous White British genetic ancestry profiles (Byroft et al., 2018). Results will be presented, parallel to the primary results in the main report body, in the Supplemental Materials. Any discrepancies will be noted (that is, any places where the conclusions with regard to the three research questions differ between the full and White British ancestry sub-sample analyses); however, our primary interest and interpretation will be on the results from the full, diverse sample. 


\section{Funding}

This project is supported by a grant from the John Templeton Foundation (JTF58792). 


\section{References}

Abdolmaleky, H. M., Smith, C. L., Faraone, S. V., Shafa, R., Stone, W., Glatt, S. J., \& Tsuang, M. T. (2004). Methylomics in psychiatry: modulation of gene-environment interactions may be through DNA methylation. American Journal of Medical Genetics Part B: Neuropsychiatric Genetics, 127(1), 51-59.

Allen, H. L., Estrada, K., Lettre, G., Berndt, S. I., Weedon, M. N., Rivadeneira, F., ... \& Ferreira, T. (2010). Hundreds of variants clustered in genomic loci and biological pathways affect human height. Nature, 467(7317), 832.

Barcellos, S. H., Carvalho, L. S., \& Turley, P. (2018). Education can reduce health differences related to genetic risk of obesity. Proceedings of the National Academy of Sciences, 115(42), E9765-E9772.

Belsky, J., Bakermans-Kranenburg, M. J., \& Van IJzendoorn, M. H. (2007). For better and for worse: Differential susceptibility to environmental influences. Current directions in psychological science, 16(6), 300-304.

Berg, J. J., Harpak, A., Sinnott-Armstrong, N., Joergensen, A. M., Mostafavi, H., Field, Y., ... \& Coop, G. (2019). Reduced signal for polygenic adaptation of height in UK Biobank. eLife, 8, e39725.

Border, R., Johnson, E. C., Evans, L. M., Smolen, A., Berley, N., Sullivan, P. F., \& Keller, M. C. (2019). No Support for Historical Candidate Gene or Candidate Gene-by-Interaction Hypotheses for Major Depression Across Multiple Large Samples. American Journal of Psychiatry, in press.

Boyce, W. T., \& Ellis, B. J. (2005). Biological sensitivity to context: I. An evolutionary-developmental theory of the origins and functions of stress reactivity. Development and psychopathology, 17(2), 271-301. 
Briley, D. A., Livengood, J., \& Derringer, J. (2018). Behaviour genetic frameworks of causal reasoning for personality psychology. European Journal of Personality, 32(3), 202-220.

Briley, D. A., Livengood, J., Derringer, J., Tucker-Drob, E. M., Fraley, R. C., \& Roberts, B. W. (2019). Interpreting behavior genetic models: seven developmental processes to understand. Behavior genetics, 49(2), 196-210.

Bulik-Sullivan, B., Finucane, H. K., Anttila, V., Gusev, A., Day, F. R., Loh, P. R., ... \& Daly, M. J. (2015). An atlas of genetic correlations across human diseases and traits. Nature genetics, 47(11), 1236.

Bycroft, C., Freeman, C., Petkova, D., Band, G., Elliott, L. T., Sharp, K., ... \& Cortes, A. (2018). The UK Biobank resource with deep phenotyping and genomic data. Nature, 562(7726), 203-209.

Caspi, A., McClay, J., Moffitt, T. E., Mill, J., Martin, J., Craig, I. W., ... \& Poulton, R. (2002). Role of genotype in the cycle of violence in maltreated children. Science, 297(5582), 851-854.

Caspi, A., Sugden, K., Moffitt, T. E., Taylor, A., Craig, I. W., Harrington, H., ... \& Poulton, R. (2003). Influence of life stress on depression: moderation by a polymorphism in the 5-HTT gene. Science, 301(5631), 386-389.

Celis-Morales, C. A., Lyall, D. M., Gray, S. R., Steell, L., Anderson, J., Iliodromiti, S., ... \& Bailey, M. E. (2017). Dietary fat and total energy intake modifies the association of genetic profile risk score on obesity: evidence from 48170 UK Biobank participants. International Journal of Obesity, 41(12), 1761.

Champely, S. (2018). pwr: Basic Functions for Power Analysis. R package version 1.2-2.

\section{https://CRAN.R-project.org/package=pwr}

Chabris, C. F., Lee, J. J., Cesarini, D., Benjamin, D. J., \& Laibson, D. I. (2015). The fourth law of behavior genetics. Current Directions in Psychological Science, 24(4), 304-312. 
Csardi, G., \& Nepusz, T. (2006). The igraph software package for complex network research. InterJournal, Complex Systems, 1695(5), 1-9. http://igraph.org

Derringer, J. (2018, April 16). A simple correction for non-independent tests. https://doi.org/10.31234/osf.io/f2tyw (pre-print, under review).

Dick, D. M., Agrawal, A., Keller, M. C., Adkins, A., Aliev, F., Monroe, S., ... \& Sher, K. J. (2015). Candidate gene-environment interaction research: Reflections and recommendations. Perspectives on Psychological Science, 10(1), 37-59.

Duncan, L. E., \& Keller, M. C. (2011). A critical review of the first 10 years of candidate gene-by-environment interaction research in psychiatry. American Journal of Psychiatry, 168(10), 1041-1049.

Flint, E., \& Cummins, S. (2016). Active commuting and obesity in mid-life: cross-sectional, observational evidence from UK Biobank. The lancet Diabetes \& endocrinology, 4(5), 420-435.

Haberstick, B. C., Lessem, J. M., Hewitt, J. K., Smolen, A., Hopfer, C. J., Halpern, C. T., ... \& Williams, R. B. (2014). MAOA genotype, childhood maltreatment, and their interaction in the etiology of adult antisocial behaviors. Biological psychiatry, 75(1), 25-30.

Hill, W. D., Hagenaars, S. P., Marioni, R. E., Harris, S. E., Liewald, D. C., Davies, G., ... \& Deary, I. J. (2016). Molecular genetic contributions to social deprivation and household income in UK Biobank. Current Biology, 26(22), 3083-3089.

Hsu, L., Jiao, S., Dai, J. Y., Hutter, C., Peters, U., \& Kooperberg, C. (2012). Powerful cocktail methods for detecting genome-wide gene-environment interaction. Genetic epidemiology, 36(3), 183-194. 
Karg, K., Burmeister, M., Shedden, K., \& Sen, S. (2011). The serotonin transporter promoter variant (5-HTTLPR), stress, and depression meta-analysis revisited: evidence of genetic moderation. Archives of general psychiatry, 68(5), 444-454.

Keller, M. C. (2014). Genex environment interaction studies have not properly controlled for potential confounders: the problem and the (simple) solution. Biological psychiatry, 75(1), 18-24.

Kendler, K. S., Aggen, S. H., Knudsen, G. P., Røysamb, E., Neale, M. C., \& Reichborn-Kjennerud, T. (2011). The structure of genetic and environmental risk factors for syndromal and subsyndromal common DSM-IV axis I and all axis II disorders. American Journal of Psychiatry, 168(1), 29-39.

Klengel, T., \& Binder, E. B. (2015). Epigenetics of stress-related psychiatric disorders and genex environment interactions. Neuron, 86(6), 1343-1357.

Lyall, D. M., Cullen, B., Allerhand, M., Smith, D. J., Mackay, D., Evans, J., ... \& Pell, J. P. (2016). Cognitive test scores in UK Biobank: data reduction in 480,416 participants and longitudinal stability in 20,346 participants. PloS one, 11(4), e0154222.

Maher, B. (2008). Personal genomes: The case of the missing heritability. Nature News, 456(7218), 18-21.

Marsaglia, G., Tsang, W. W., \& Wang, J. (2003). Evaluating Kolmogorov's distribution. Journal of Statistical Software, 8(18), 1-4.

Mills, M. C., \& Rahal, C. (2019). A scientometric review of genome-wide association studies. Communications biology, 2(1), 9.

Mirisola, A. \& Seta, L. (2016). pequod: Moderated Regression Package. R package version 0.0-5. https://CRAN.R-project.org/package=pequod 
Nyholt, D. R. (2004). A simple correction for multiple testing for single-nucleotide polymorphisms in linkage disequilibrium with each other. The American Journal of Human Genetics, 74(4), 765-769.

Peyrot, W. J., Van der Auwera, S., Milaneschi, Y., Dolan, C. V., Madden, P. A., Sullivan, P. F., ... \& Mullins, N. (2018). Does childhood trauma moderate polygenic risk for depression? A meta-analysis of 5765 subjects from the Psychiatric Genomics Consortium. Biological psychiatry, 84(2), 138-147.

R Core Team (2018). R: A language and environment for statistical computing. R Foundation for Statistical Computing, Vienna, Austria. URL https://www.R-project.org/.

Rietveld, C. A., Medland, S. E., Derringer, J., Yang, J., Esko, T., Martin, N. W., ... \& Albrecht, E. (2013). GWAS of 126,559 individuals identifies genetic variants associated with educational attainment. Science, 340(6139), 1467-1471.

Ripke, S., Sanders, A. R., Kendler, K. S., Levinson, D. F., Sklar, P., Holmans, P. A., ... \& Scolnick, E. (2011). Genome-wide association study identifies five new schizophrenia loci. Nature genetics, 43(10), 969.

Risch, N., Herrell, R., Lehner, T., Liang, K. Y., Eaves, L., Hoh, J., .. \& Merikangas, K. R. (2009). Interaction between the serotonin transporter gene (5-HTTLPR), stressful life events, and risk of depression: a meta-analysis. Jama, 301(23), 2462-2471.

Roisman, G. I., Newman, D. A., Fraley, R. C., Haltigan, J. D., Groh, A. M., \& Haydon, K. C. (2012). Distinguishing differential susceptibility from diathesis-stress: Recommendations for evaluating interaction effects. Development and psychopathology, 24(2), 389-409.

Saltz, J. B., Bell, A. M., Flint, J., Gomulkiewicz, R., Hughes, K. A., \& Keagy, J. (2018). Why does the magnitude of genotype-by-environment interaction vary?. Ecology and Evolution. 
Schröder, P. C., Li, J., Wong, G. W., \& Schaub, B. (2015). The rural-urban enigma of allergy: What can we learn from studies around the world?. Pediatric Allergy and Immunology, 26(2), 95-102.

Smith, G. D., Whitley, E., Dorling, D., \& Gunnell, D. (2001). Area based measures of social and economic circumstances: cause specific mortality patterns depend on the choice of index. Journal of Epidemiology \& Community Health, 55(2), 149-150.

Sohail, M., Maier, R. M., Ganna, A., Bloemendal, A., Martin, A. R., Turchin, M. C., ... \& Neale, B. (2018). Signals of polygenic adaptation on height have been overestimated due to uncorrected population structure in genome-wide association studies. bioRxiv, 355057.

Townsend, P. (1987). Deprivation. Journal of social policy, 16(2), 125-146.

Tucker-Drob, E. M., \& Bates, T. C. (2016). Large cross-national differences in genex socioeconomic status interaction on intelligence. Psychological science, 27(2), 138-149.

Van Os, J., Rutten, B. P., \& Poulton, R. (2008). Gene-environment interactions in schizophrenia: review of epidemiological findings and future directions. Schizophrenia bulletin, 34(6), 1066-1082.

Vassos, E., Agerbo, E., Mors, O., \& Pedersen, C. B. (2016). Urban-rural differences in incidence rates of psychiatric disorders in Denmark. The British Journal of Psychiatry, 208(5), 435-440.

Yang, J., Weedon, M. N., Purcell, S., Lettre, G., Estrada, K., Willer, C. J., ... \& Mägi, R. (2011). Genomic inflation factors under polygenic inheritance. European Journal of Human Genetics, 19(7), 807-812.

Zeilinger, S., Kühnel, B., Klopp, N., Baurecht, H., Kleinschmidt, A., Gieger, C., ... \& Strauch, K. (2013). Tobacco smoking leads to extensive genome-wide changes in DNA methylation. PloS one, 8(5), e63812. 